\title{
Towards Eco-Friendly Home Networking
}

\author{
Mathias Gibbens, Chris Gniady and Beichuan Zhang \\ Department of Computer Science \\ The University of Arizona \\ Tucson, Arizona \\ \{gibmat, gniady,bzhang\}@es.arizona.edu
}

\begin{abstract}
Home networking is becoming increasingly sophisticated as users connect ever more networked devices. In the past, home networks typically consisted of a simple router and maybe a few computers. Now, high speed wireless networks are commonplace in homes with a large number of devices: TVs, game consoles, home theater systems, and even home automation systems. This is in addition to standard computing devices such as desktops, laptops, tablets, smart phones and network-attached storage. Smart homes with many wireless sensors to improve quality of life are also emerging. However, home network devices such as routers and broadband modems have been designed for maximum performance with limited consideration of energy optimization when the devices are idle or serving low bandwidth traffic. The goal of this paper is to analyze network activity of wireless home routers, investigate opportunities for energy saving, and present mechanisms for improving the energy efficiency of wireless home routers. We analyzed five week-long traces of home network traffic and identified a number of energy saving opportunities. Through detailed trace-based simulation and implementation measurements we are able to reduce the wireless energy consumption of the home router by $12-59 \%$ while incurring only minor delay of the initial packet delivery after leaving the low energy state. Modification of an actual router's behavior shows that the proposed energy optimizations are feasible with existing clients.
\end{abstract}

Keywords-home networks; energy efficiency

\section{INTRODUCTION}

Energy efficiency has become a critical issue in all aspects of computing, from large data centers and the Internet to cellphones and home devices. According to the SMART2020 report [1], information and communication technologies consumed hundreds of terawatt-hours of energy in the United States in 2006, which cost billions of dollars and generated a $\mathrm{CO}_{2}$ footprint similar to that of the aviation industry. While data centers and large telecom infrastructures have high power densities, we cannot ignore the growing home networking infrastructure and the large number of these networks. Though individual home networks may not be seen as a significant energy consumer, the vast number of households with growing network infrastructure can account for a sizable fraction of energy consumption in the United States. A 2013 report states that there are 122 million housing units in the United States and that 88 million of them have a high speed broadband connection [2]. Assuming each broadband connected household has a single modern wireless router such as the Asus RTAC66U with a measured power consumption of 9.7 watts [3], we can estimate energy consumption in the United States due to home networking to be roughly $7,480 \mathrm{GWh}$ per year. For comparison, this much energy could power 690 average US households for an entire year [4].

This number may not seem alarming right now, but it will be in the near future given current trends in home networking. First, individual home networks are becoming more sophisticated, resulting in home routers consuming more and more power as we demand higher performance and functionality. Furthermore, there is a wide range of power demand among home routers as shown in a recent comparison [5]. Second, the number of home networks is growing quickly worldwide. In the United States, the federal government has invested $\$ 7.2$ billion to improve broadband infrastructure via the American Recovery and Reinvestment Act. In parallel, the Federal Communications Commission is defining a national strategy to improve national broadband coverage and quality. The United States, however, is only ranked 15th in terms of broadband penetration in the world. Thus, if we consider all home networks in the entire world, improving their energy efficiency would have significant positive impacts on our environment.

Manufacturers are striving to make their networking components more energy efficient and many home networking components are labeled with an energy star rating. However, an energy star rating does not necessarily imply that networking equipment is operating in the most efficient power state. Currently, home routers operate at full power even if there are no devices active on the network, in order to guarantee high performance and good connectivity. This approach is not very energy efficient, as the networking devices in an idle state are not taking advantage of powering down their internal components to reduce energy consumption.

Home network activity can vary and can offer different opportunities for energy optimizations. Subsequently, we investigate home network traffic patterns and explore the opportunity for energy efficiency optimizations. We make the following contributions in this paper: (1) trace and evaluate several household traffic patterns to identify opportunities for energy optimization, (2) propose different energy management techniques to expose the challenges of energy management in home networking, and (3) identify requirements for future research in home networking efficiency. 


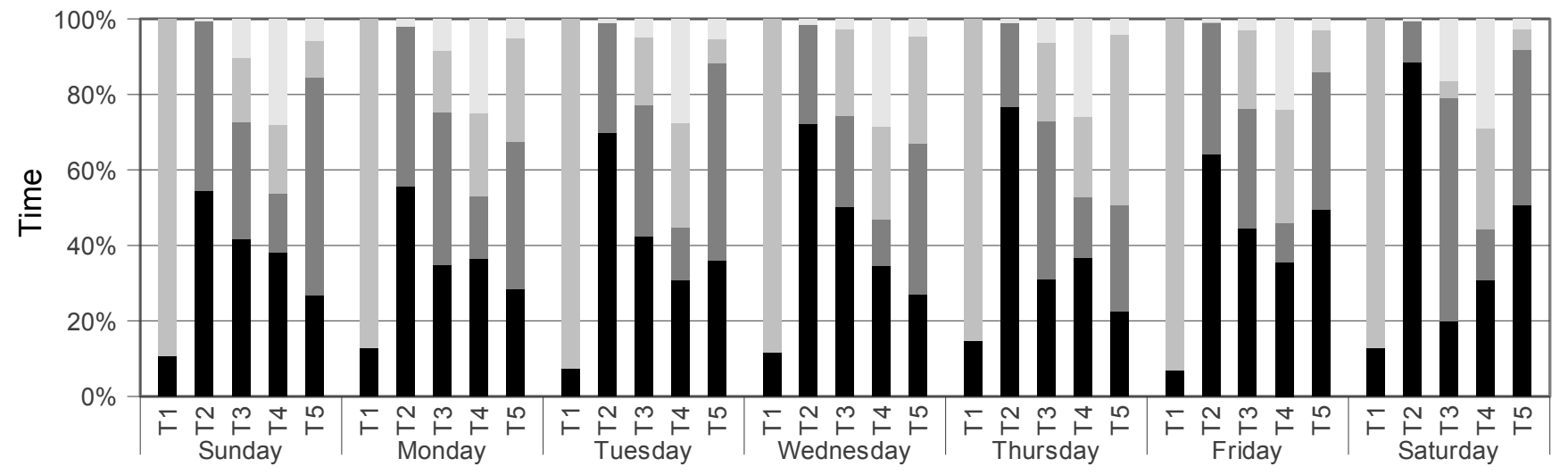

Fig. 1. Wireless network activity in home routers.

\section{Motivation}

Consumer routers today resemble minicomputers that can run a basic operating system, have a significant amount of memory, and can utilize attached devices, like hard drives or printers, in addition to providing high bandwidth wired and wireless interfaces. The router is responsible for routing traffic between home devices and the Internet, as well as optionally providing many other functionalities such as a DLNA multimedia server, a file server, a firewall and so on. Households are also becoming more and more connected. Most multimedia devices (TV, Blu-ray players, gaming consoles, etc.), smart appliances, and security systems require some sort of network connectivity for full functionality. This is in addition to mobile phones, tablets, and portable computers which have become commonplace. While most of these devices require network connectivity, many households are not prewired for network connections. As a result, wireless connectivity has become a very popular option for providing a flexible networking infrastructure. As the number of wireless-enabled devices continues to grow, the prevalence of wireless networks will increase to satisfy demand.

While a wireless connection is convenient, the wireless interfaces need to provide high bandwidth to satisfy the demand of all connected devices, which increases design complexity and power demand of the router. Modern wireless routers have been found to consume 4-11 watts, depending on what features are enabled and the wireless specification provided [3], [5]. Generally speaking, newer 802.11AC-type wireless networks will consume more energy than the older 802.11N-type networks.

A significant amount of research has focused on the power consumption of large Internet routers which consist of multiple chassis each containing multiple line cards that have over provisioned connections to provide performance and reliability. In this scenario, the line cards can be simply turned off when links are not used [6]. Even in home networking, wired network interfaces can be powered down when inactive, and the router utilizes a link sense signal to detect the presence
TABLE I. WIRELESS TRACE CHARACTERISTICS

\begin{tabular}{|c||c|c|c|c|c|}
\hline Trace & T1 & T2 & T3 & T4 & T5 \\
\hline \hline Average concurrent devices & 1 & 5 & 4 & 4 & 2 \\
Maximum concurrent devices & 1 & 9 & 7 & 6 & 3 \\
Initial associations & 13 & 16 & 14 & 31 & 35 \\
Average time with no client [h] & 10.7 & 0.08 & 2.07 & 1.32 & 0.92 \\
Traffic volume [GB] & 5.57 & 40.21 & 4.22 & 3.52 & 12.97 \\
\hline
\end{tabular}

of a client on the network. Wireless network interfaces, on the other hand, cannot be easily powered down because the router needs to broadcast its presence and listen for any clients that will attempt to connect over the shared medium of the wireless signal. However, long periods of idle time, both with and without any clients present, can offer energy saving opportunities if properly realized.

\section{A. Potential for energy optimization}

Before we consider any optimizations, we need to understand the frequency and type of traffic that is seen in personal home networks. While there are existing databases of wireless traces for research purposes, such as CRAWDAD [7], they do not contain the details that would help us understand the traffic patterns between devices or study optimizations in a home network setting. To incorporate all traffic details that would allow us a detailed trace driven analysis of home network traffic, we collected network traces from five diverse households: single occupants, many roommates sharing an apartment and individual families. The traffic was captured using tcpdump over a period of one week from modified home routers. The volunteers were instructed to use their network as they would normally to help ensure a representative trace from each household. To protect the privacy of the users and reduce the capture file size, only the headers of each packet were saved. These headers contain information such as IP addresses, a timestamp, and total packet size, but not actual data like URLs or form submissions.

Figure 1 presents the wireless network utilization for each distinct trace over the week-long period (labeled T1-5). The activity was categorized into one of four groups: active client 
periods when there is active communication between the router and the wireless clients; idle client periods when the clients are connected but there is no network communication; no client idle periods when there are no wireless clients connected; and finally, no client broadcast periods when there is broadcast activity, originating from the wired network, present on wireless interfaces but there are no clients to receive these packets. Table I complements the statistics from Figure 1 and shows additional information about each week-long trace: the average number of simultaneously active wireless devices, the maximum number of devices seen at a single time over the whole trace, the number of initial associations that occurred when no other wireless clients were present, the average length of idle times when no clients were present, and the total traffic volume.

Trace 1 came from a network which is mostly idle for long periods of time, making it ideal for potential energy savings. There was a single computer present on this wireless network that was on a distinct subnet from the wired network, so the broadcast traffic from the wired devices is not present on the wireless interface. Traces 2 and 3 demonstrate the personal wireless networks of households with several occupants that heavily utilize the network for video streaming and online gaming resulting in very short idle times seen at the wireless router, potentially precluding some possible optimizations. Trace 4 is from a single family with several devices on the network, including streaming media and smart appliances. In contrast to Trace 1, this wireless network shares the same subnet as the wired one, and constantly receives broadcast traffic, such as UPnP messages and status updates, even when no one is home. Finally, Trace 5 is from a single occupant household with heavy network activity due to several network file systems mounted on the machine which were accessed while working.

Traffic in home networks tends to be bursty, unlike more uniform network activity that is seen in larger business networks or Internet routing links. For example, consider what happens when a person loads a single web page in their browser. A flurry of activity is initiated in a very short period of time: First, their computer may have to issue an ARP request to locate the router on the local network. Second, a DNS lookup will be performed to transform the domain the person typed into an IP address. Third, one or more HTTP sessions are established to actually load the content which was requested. As the main page is loaded, additional resources like images or JavaScript will be fetched as well, potentially causing more DNS queries to be issued. Finally, the person will read the web page, and their network will be idle again.

Figure 2 shows that four of the five home network traces clearly exhibit this bursty network activity (notice that the yaxis is logarithmic). Trace 4 also shows burstyness, but it is not as dramatically as the other traces. For each trace, the inter-arrival time between each packet was measured, then formed into a distribution based on one second binning. The vast majority of packets seen in every trace occurred within one second of the preceding one. Once the inter-arrival time

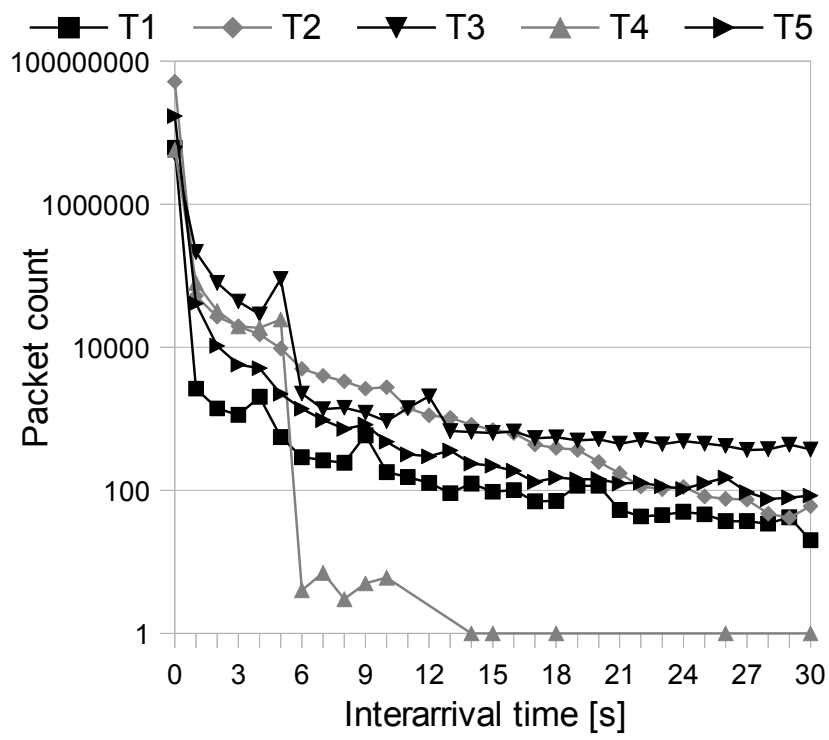

Fig. 2. Distribution of bursty traffic in each trace.

reaches 15 seconds, the distribution begins to trail off. Interarrival times of more than 30 seconds are not shown, but follow the same general downward slope for each trace.

In a network that exhibits more uniform traffic, the curve shown in Figure 2 would be steeper and be more closely asymptotic to both axes of the graph. Not all traffic in a home network is bursty in nature, such as video streaming or VoIP, and this accounts for the majority of packets with very small inter-arrival times.

From Figures 1 and 2 and Table I, we can make several observations about these home networks. First, the "no client" fraction of time is surprisingly small, except in T1. One may expect that a typical user or users are away from their home or asleep for a majority of the time and that the idle periods should be much longer. However, the network is only truly idle on average for 4.8 hours a day for Traces $2-5$, which have more sophisticated traffic patterns than the partitioned network in T1. Second, broadcast traffic that is sent out through the wireless interface when no clients are connected consumes a significant portion of the time. This case clearly ignores any efficiency ideas as there are no clients to receive this broadcast traffic. Third, there is a significant portion of the time when clients are connected to the access point but there is no network activity. Fourth, traffic patterns in these networks consist of periods of very heavy activity interspersed with much lighter ones. Finally, there is not much difference for individual households with respect to the time distribution between the activities of the wireless interfaces.

\section{B. Characteristics of a home router}

Figure 3 shows the power states of the wireless radios of an Asus RT-N16 WiFi-N router running the DD-WRT firmware [8], which was the primary router used in this research. For our measurements, we set the network mode 


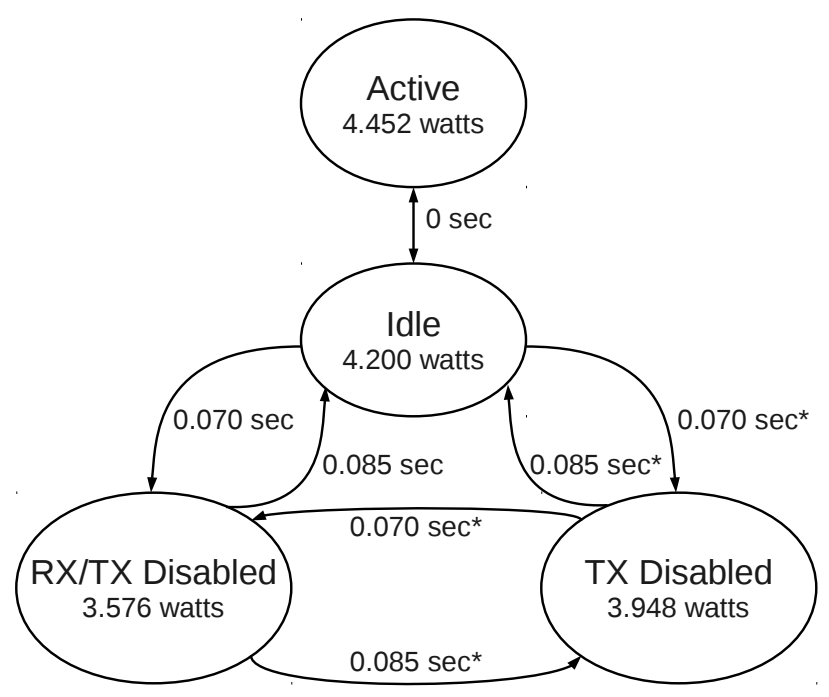

Fig. 3. Power states of the wireless router.

to "N" and left all other settings at their defaults. To profile the router, we used a National Instruments PCI-6230 Data Acquisition card (NI PCI-6230) combined with the National Instruments Signal Express measuring software. We measured the power associated with the radio transmission by disabling the radios, enabling them and idling, and actively transmitting data over the network. The router was powered by an instrumented power supply and the instantaneous current draw was calculated by capturing the voltage drop across a $10 \mathrm{~m} \Omega$ current sense resistor wired into the router's power supply connection. Measured power consumption was obtained as an average over five minutes of sampling in each state.

The router's hardware and/or driver does not support individual control of the transmit and receive radios, so we estimate that the transmit power is $60 \%$ of total radio power [9], [10]. Transition from an active state to idle and back to active is instantaneous since no change in hardware state is required and any pending packets can be transmitted immediately. Shutting down transmit and receive radios takes on average $70 \mathrm{~ms}$ and it takes on average $85 \mathrm{~ms}$ to turn them back on before any packet is transmitted. Since we are unable to individually measure the shutdown and power up of just the transmit radio, we assumed that it takes the same amount of time to transition between the resulting power states.

We observe in Figure 3 that radios are significant energy consumers in home routers; however, completely powering down the access point antennas has significant ramifications. First, if power management is achieved by periodically shutting down the wireless radio when the clients are connected, their connectivity may be severed. Second, when the router's wireless ratios are powered down, arriving clients may not be able to detect the presence of the router and therefore will not be able to connect. Therefore, a more advanced radio modulation that considers client activity is required to provide high performance and maintain good connectivity while reducing the energy consumption of the router.

\section{DESIGN}

We will approach the optimization of the router's wireless energy in four steps. First, we maximize the idle times when no clients are connected. Second, we exploit these longer idle times with no clients to improve energy efficiency when the router is not being used. Third, we explore mechanisms to optimize energy efficiency when clients are associated to the network but temporarily not communicating. Finally, we propose a more unified approach that aggressively optimizes energy for periods with or without clients. All of these strategies for reducing the energy consumption of a wireless router need to preserve existing wireless protocols, which will allow for the transparent optimization of energy efficiency in various scenarios without requiring modification of the client's hardware or software.

\section{A. Absence of WiFi clients}

While the absence of wireless clients should indicate that there is no wireless traffic present and that the antennas can be safely powered down, this is not the case as we can see from Figure 1. Specifically, in the traces there is a significant amount of broadcast traffic present on the wireless network even when there are no clients connected. This traffic, generated by protocols such as ARP, IP broadcast/multicast, DHCP, and UPnP, is important and necessary for clients to accept and respond to. However, when there are no wireless clients connected there is no need for the broadcast of this traffic as there is no one to receive nor respond to it.

Therefore, a simple optimization of filtering out all wireless traffic when no clients are connected will extend the idle periods for the wireless antennas. This filtering can be easily done with little to no overhead as the router already knows that no wireless clients are present and it can therefore drop broadcast packets via a simple network routing rule. While eliminating these broadcasts can result in some minor improvements in energy efficiency, since the energy due to transmissions is eliminated, the gains are not dramatic as the router's radios remain on. However, longer unified idle periods can potentially enable us to power down the network interface during those periods of inactivity for greater improvements in energy efficiency.

Because the router will not have to worry about handling wireless traffic, idle periods without clients present offer the best opportunity to save energy by powering down the radios. Ideally, the router would power down its radios upon the last client's departure and power them back on when a client arrives. The challenge is that clients can arrive at any time and without active radios the router will not be able to announce its presence nor respond to a client's association requests. Under normal operation, the access point will periodically transmit beacons that advertise the wireless network's presence as shown in Figure 4. A client must see the BSSID beacon before initiating the attempt to establish a wireless connection. If the 


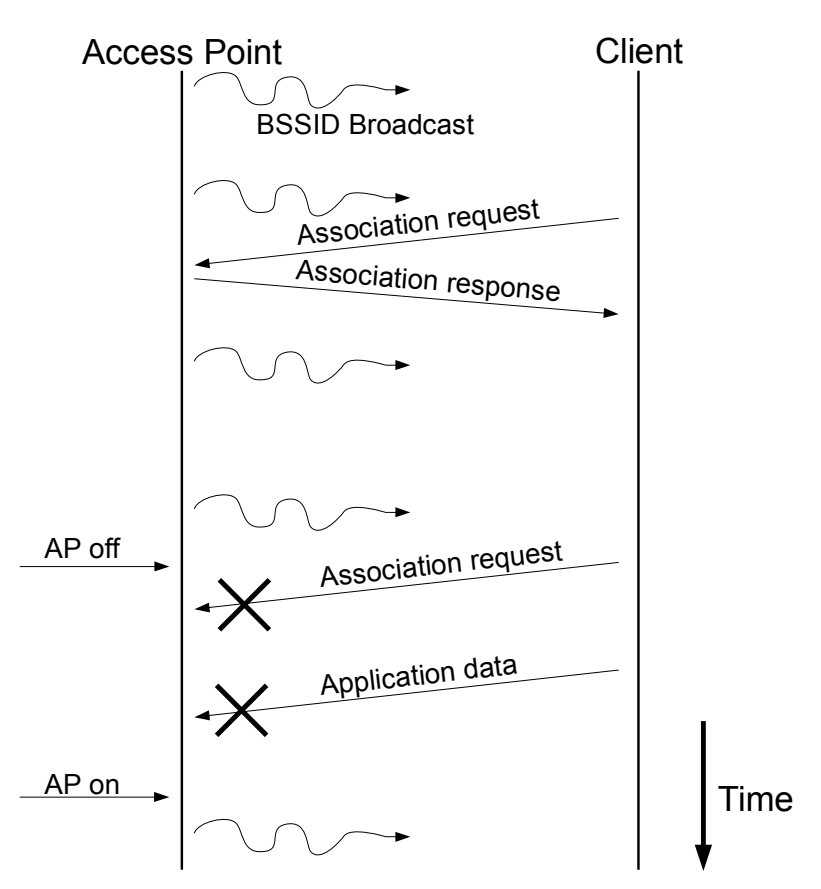

Fig. 4. Interaction of an access point and client.

radios are shut down before the client starts the association process, the association will fail as we can see in Figure 4.

To accommodate the arrival of a new client, completely powering down the radios for an extended amount of time is not possible. We must power cycle the radios on and off periodically to transmit the router's presence and accept new connections from arriving clients. We need to consider the trade off between energy savings and potential delays that a user will encounter when attempting to associate to the network. In addition, the association process is not instantaneous and it can take few seconds before the client begins an attempt to associate. This delay was observed to be the result of the client network software queuing information about changes to the available networks and only updating this information periodically. Since there is already some expected delay when associating, a well-designed approach to powering down the radios to reduce energy consumption when no clients are connected could be implemented without adversely affecting the users.

\section{B. Power cycling parameters}

Careful selection of the parameters of when to start power cycling and how long to keep the radios on and off is critical to minimize the negative impact on the user while conserving energy. While a simple approach would start power cycling immediately following the last client's departure, short disassociated periods due either to the rebooting of a device or wireless interference were seen in some traces. Since the goal of our approach is to address the long idle periods when users are away, short periods should not trigger the power cycling mode to minimize unnecessary delays for the clients that are temporarily unassociated but have not actually left. To account
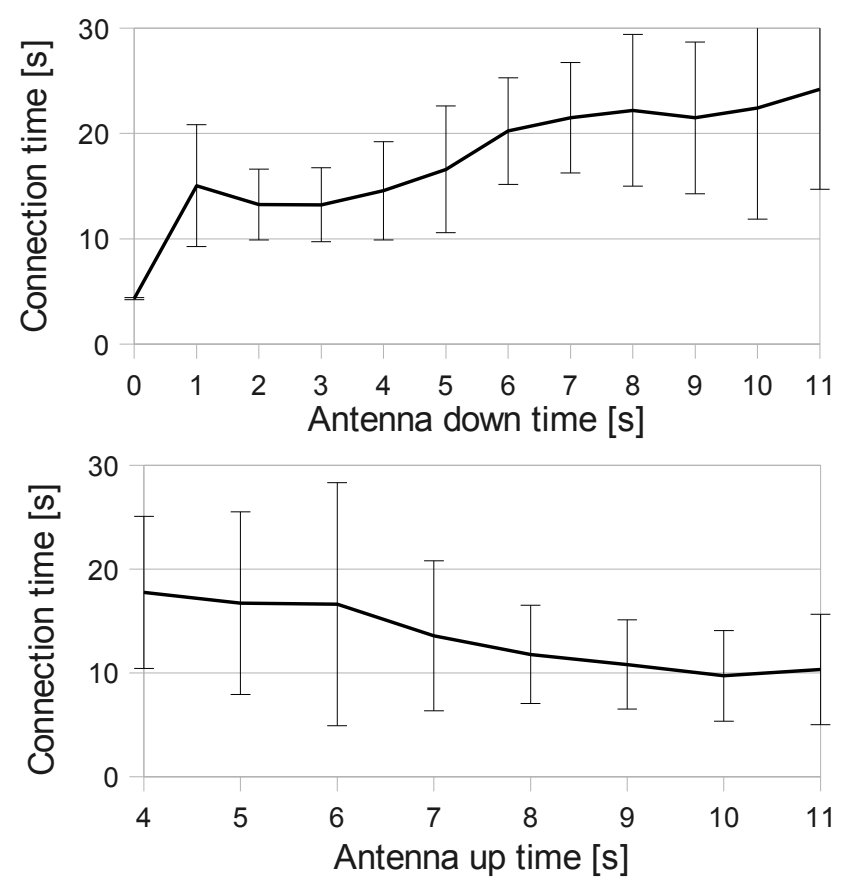

Fig. 5. Average time to full network connectivity when cycling radios.

for such short periods, we set the timeout to 30 seconds after the last client departs before we start power cycling the radios.

Once the power cycling is active, the arriving client will encounter a delay before it sees the BSSID being transmitted. Once the BSSID is observed by the client it will attempt to associate to the access point and the access point needs to remain active long enough to provide a successful connection. Figure 5 shows how the length of time that radios are enabled or disabled impacts the average time required for an initial client to fully associate with the access point and achieve network connectivity. In each graph, we varied only the up or down time of the Asus RT-N16 router; the other time was fixed at five seconds. The fixed time was selected because initial tests showed that the wireless interface must be active for at least four seconds for a client to reliably connect to the access point. Error bars show one standard deviation from the average time.

The results in Figure 5 follow the intuitive idea that shortening the length of time that the radios are on results in longer delays as the clients have less time to notice the BSSID and successfully initiate the connection. Similarly, lengthening the time the radios are off makes the client wait longer before the router presence is announced and the client observes it. An intriguing behavior occurs when we increase radio off time from 1 to 2 seconds. The decrease in connection time while increasing the down time of the router's radios was caused by two cycles: the router turning its radios on and off, and the client periodically scanning for available access points. The network manager on the client scans for the presence of access points every 3-5 seconds and increasing the period to 2 seconds resulted in the cycles of beaconing by the router and scanning by the client aligning, allowing for faster connection 
than at one second intervals. The cycles remained aligned until about the four second mark, at which point the connection time begins to significantly increase again.

Considering Figure 5, the minimum time required for a client to associate and the behavior of a client scanning for available networks, we selected a five second on, five second off radio cycle for the router to employ when clients are not present. This 50/50 split will allow the router to be in a lower power mode for up to half the time while not causing excessive delay when a client wishes to connect. The power cycling of the radios is performed by using the wireless API present in the Linux kernel [11], and exposed via utilities like iwconfig.

\section{Presence of WiFi clients}

Powering down a router's radios when wireless clients are present is problematic as it can introduce delays during application communication or even cause client disconnects, directly impacting the user. However, there is a significant amount of time when clients are associated to the network but not actively transmitting data as we can see in Figure 1. Those long idle times present the potential for energy optimizations. If we address them by the duty cycling idea described earlier, we would like to power the radios on and off while preventing the client from disconnecting and the user seeing a "network unreachable" error. In order to find the length of time that a client will tolerate before disassociating, we measured devices running Android 4.1, Debian 7, and Windows 7 and found the average time to be 7,10 , and 9 seconds, respectively.

As the wireless medium is prone to interference, a client's hardware will usually attempt $4-7$ transparent retransmissions of any packet that was not properly sent or acknowledged in time before informing the software network stack of a transmission failure [12]. The software stack may also attempt retransmission, if for example a TCP connection is in use; ultimately, however, if normal operation is interrupted for too long the user will see the network error.

While these delays give us some opportunity to power cycle before causing network interruptions, the power cycling delays are being directly introduced into user interaction with the applications, negatively affecting the user experience. To eliminate delays being exposed to the user, while the user is connected and using the network, we rely on the key observation that the router does not initiate activity, so its responsiveness is not impacted as long as the router can immediately receive and process user requests. Therefore, the router can power down the transmit antennas, while keeping the receive antennas on to listen for the arrival of new requests from clients.

As we can see in Figure 3 the transition between the transmit antenna being disabled and then active takes only $85 \mathrm{~ms}$. However, this delay can be completely hidden from the user. First, the router does not initiate data transmission, and once it receives the user request it must send it to a remote server before it needs to reply with the data to the user. This gives time to initiate powering on transmit antennas

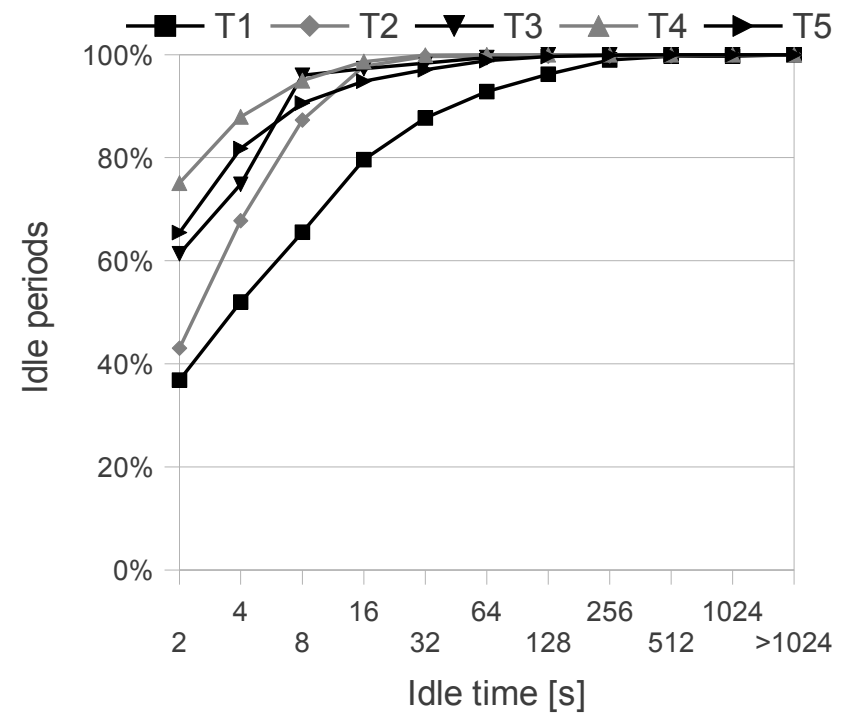

Fig. 6. Cumulative distribution of idle time lengths when clients are associated.

as soon as the router processes a request from the wireless client. Second, any remaining transition delay can be hidden within the user's perception time, as any tasks that are less than $100 \mathrm{~ms}$ in duration appear instantaneous to humans [13].

While we can be quite aggressive in powering down the transmit antennas, short periods offer very little opportunity for energy savings as it costs both time and energy to power cycle the antennas. Therefore, any idle time we wish to exploit for energy optimizations needs to be long enough to offset the energy associated with power cycling. This limit is called the break-even time, and is found by solving for time in an equation that balances energy consumption under normal operation and the energy required by switching to and consumed in a lower power mode. Using worst case assumptions, the break-even time for transitioning the transmit antenna on the Asus RT-N16 router is 1.5 seconds: any period shorter than this would not benefit from energy savings. Figure 6 presents the cumulative distribution of idle times for each trace when at least one client is associated. We will consider only idle periods that are larger than the break-even time in our approach since they can offer the potential for energy savings. We observe that the majority of idle periods are short, with periods less than 2 seconds accounting for between $36-75 \%$ of all periods.

On the other hand, Figure 7 presents a weighted cumulative distribution of the idle times from each trace with active clients and indicates that short idle periods contribute at most $12 \%$ to the total idle time, while longer periods are actually responsible for a much larger contribution. This shows a great opportunity for energy savings while reducing switching frequency. Considering the results of Figure 7 and the ability to hide the transmit antenna transition overheads, we set the timeout for disabling the transmit radio at the break-even time of 1.5 seconds. This short timeout period will convert the majority of idle time into energy savings while eliminating 


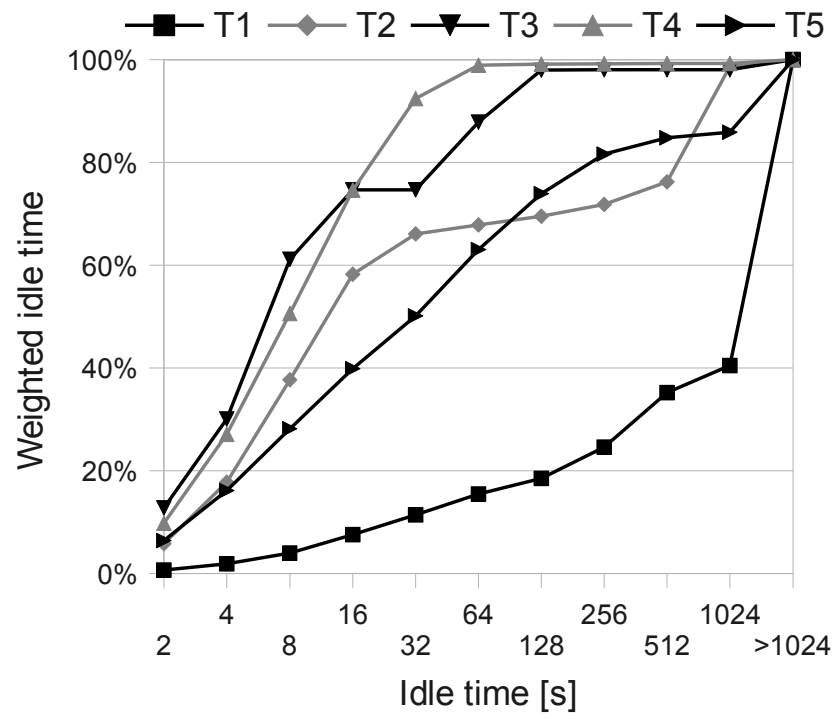

Fig. 7. Weighted cumulative distribution of idle time lengths when clients are associated.

any unnecessary switching for active communication such as streaming, video conferencing, or any other real time activities.

\section{Combining optimizations for absent and present clients}

The idea of power cycling the transmit antenna while listening for communication from clients can be extended to idle periods without clients. In our initial approach we kept the transmit antennas on and transmitting the BSSID during the five seconds that the radios were on when clients were not present. This can be further optimized by potentially shortening the transmit time of the BSSID, as beacons sent towards the end of the period are much less likely to result in a successful association attempt as it can take up to 35 seconds before a client attempts association after seeing a BSSID beacon.

Therefore, we can employ the simple optimization of transmitting the BSSID during the first portion of the on cycle and then powering the transmit radio down. This way we improve energy efficiency further, without significantly impacting the connection delays. Whenever the transmit radio is active, the receive radio must also be powered on in order to listen to association requests from the client. The receive radio remains powered on for a longer period of time to accommodate the potential delay in a client connecting. This final optimization gives us a unified approach for energy optimizations under all operating scenarios in home networking environments.

\section{Simulation Results}

To allow consistent and repeatable results we used tracebased simulations to analyze, evaluate, and compare network behavior and energy consumption under several optimization scenarios. We developed a discrete event based router simulator to processes each packet, keeping track of the time, the power state of the router, and the power switching delays. The

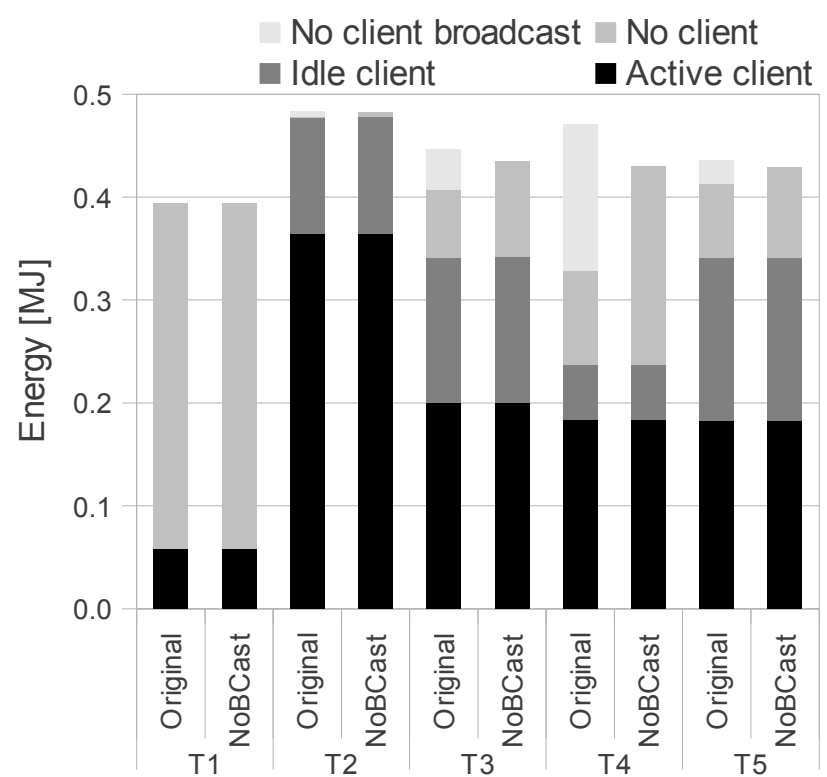

Fig. 8. Energy consumption comparison of original traces and eliminating broadcast traffic.

power and transition overheads between states were modeled according to Figure 3. The traces described in Section II were used for the evaluation of the proposed mechanisms. We also performed an implementation based evaluation in the subsequent section.

\section{A. Discarding unnecessary broadcast traffic}

Eliminating broadcast traffic when no clients are present provides a natural optimization for wireless networks. Figure 8 presents the week-long energy consumption distribution for each trace and compares the original energy consumption, corresponding to the distribution in Figure 1, to the same traffic with the broadcasts discarded when no clients are present. To focus on the energy optimizations of the wireless antennas and make it more correlated to the time the router spends in each wireless state, we have removed the base power of the router (see Figure 3) and focus solely on the radio and antenna energy in the rest of the paper. While eliminating broadcast traffic can reduce the energy consumption of the radios by as much as $9 \%$ in the case of $\mathrm{T} 4$, the average reduction is only $2.63 \%$.

As we can see from the figure, the energy savings depend on the network configuration and the number of clients and devices. The network that $\mathrm{T} 1$ was captured from is on a separate subnet, so there are no broadcasts seen from wired devices, and as a result this optimization has no effect. On the other hand, T2 has almost no idle time without clients and as a result any approach that optimizes idle time without clients will not apply. For a network to benefit from this optimization, there must be periods of time when there are no wireless clients present, such as in T3-5, yet still have active wired devices broadcasting their presence. Finally, the vast majority of this broadcast traffic in our traces originated from UPnP devices continually announcing their availability and the ARP protocol 


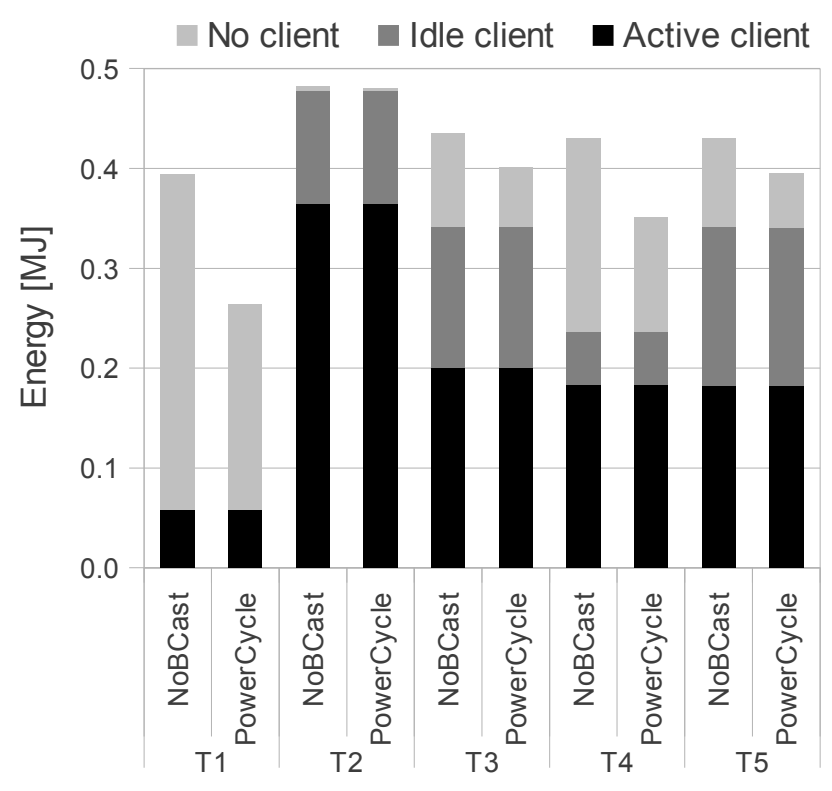

Fig. 9. Energy consumption comparison of no broadcast optimization to power cycling the radios when no clients are present.

having to respond as the local ARP cache on wired devices expired.

\section{B. Energy optimization with no clients}

Figure 9 compares the energy consumption of each trace with the broadcast traffic filtering optimization to power cycling the radios when no clients are present. As discussed in Section III-B, we use a duty cycle of five seconds on and five seconds off that balances energy savings as well as connection delays. As we can observe in Figure 1, different households have different periods of idleness without clients which translates into energy savings for the corresponding traces. T1 has the most idle time without clients and as a result has a $33 \%$ reduction in energy consumption of the radios. On the other hand, T2 has almost no idle time without clients and as a result the optimization has no impact on energy savings. Therefore, this optimization will work well for households with few devices that are not connected at all times, and will not provide much optimization for households that have a range of wireless devices that are continuously connected to the wireless network.

Power cycling the antennas will increase the connection time for the first wireless client on the network if the router happened to have its radios powered off when the client attempts to associate with the network. Figure 10 analyses the average amount of additional delay a client will observe due to power cycling of the radios in the traces. We have selected a duty cycle of five seconds on and off as the standard for our results, but for this experiment we vary the length of the off time to show the impact on the delays. This experiment corresponds to router measurements in Figure 5 and illustrates the impact on actual clients in traces.

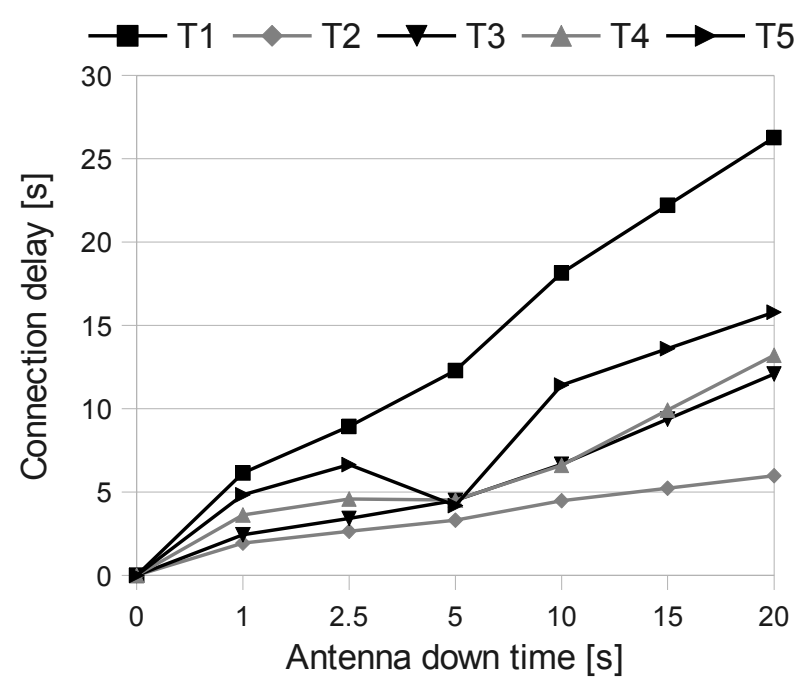

Fig. 10. Average additional time required to associate to network for increasing down times.

Trace 1 has only one wireless client and as result the client will see the worst case connection delays. The other traces have multiple clients and as result only the first client will encounter the delay and the remaining clients will not encounter any delay as the access point will be fully powered on. The first client connection delays in Traces $2-5$ will be as much as the delay shown with one client in Trace 1 , but when we amortize the connection delays among multiple clients we observe that on average the connection delays are much lower. Since homes are becoming more and more connected, we can expect that on average the additional connection delay will be low and correspond to behavior shown in Traces 2-5.

\section{Improving energy efficiency when clients are connected}

We continue evaluation by applying energy optimizations while there are clients associated. Figure 11 compares the power-cycling mechanism when no clients are present from Figure 9 to the two approaches for when clients are present: power cycling the transmit antenna when clients are present (CycleTransmit), and additionally power cycling the transmit antenna when clients are not present (CycleAll), as described in Section III-D. The timeout for power-cycling the transmit antenna was set to 1.5 seconds.

Power cycling the antennas when clients are connected but idle results in energy efficiency improvements of $10.5 \%$ on average, and a maximum of $18.3 \%$ for the studied traces. As before, the traces that benefit in case of this optimization require a significant amount of idle time with connected clients that are simply not utilizing the network. This is a common situation in households that have constantly connected clients, as seen in Traces 2-5. In this case the average savings between those 4 traces in $13.1 \%$. As before, T1 has a very different traffic pattern that either has a client connected actively using the network or the client is dissociated. Therefore, the optimizations of idle time when clients are connected do not 


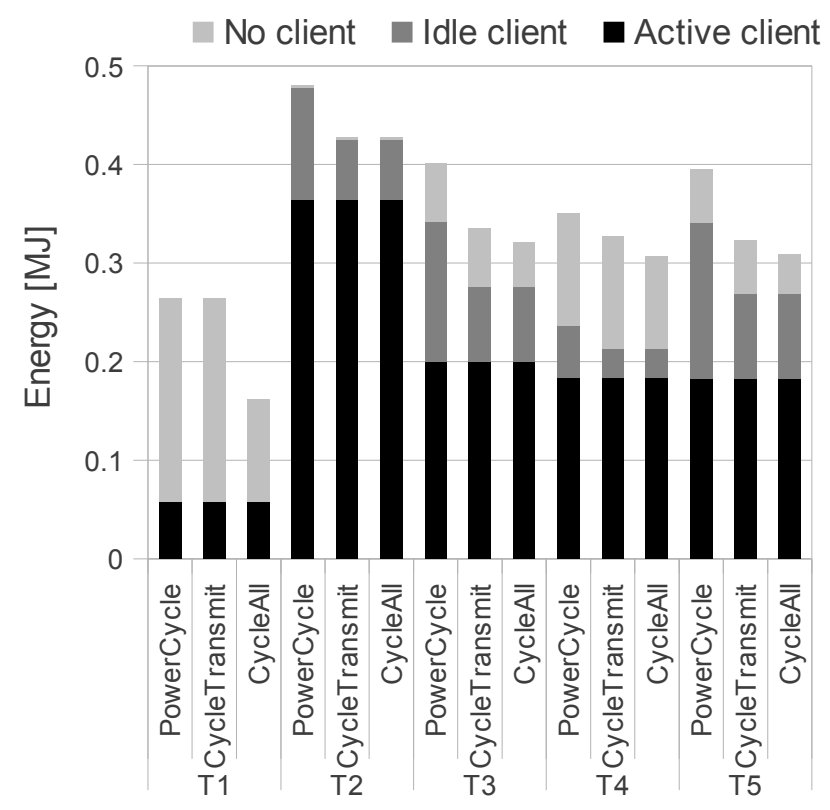

Fig. 11. Comparing energy efficiency of power cycling the transmit antennas for cases with and without clients present.

apply.

$\mathrm{T} 1$, on the other hand, will benefit significantly from further optimizations of power cycling of the radios when there are no clients connected as it has the largest no client idle time. We can see that the duty cycling of the transmit radio directly translates into energy optimizations in the router in each trace. As earlier noted, T2 with little idle time with no clients will not benefit, but on average the optimized duty cycling of the transmit antenna without clients results in an additional energy savings of $10.7 \%$ as compared to the basic PowerCycle optimization. We observe that the complementary optimizations of broadcast traffic elimination and periods with and without clients is critical to maximizing the energy efficiency of the home wireless router.

Power cycling the transmit antenna can increase delays both when clients are associating and when connected. The additional connection delay when associating is presented in Figure 12. When the transmit antenna is on for five seconds, it will be on for entire duration of the cycle and fully overlap with the receive antenna being on. The shorter periods indicate the amount of time the transmit antenna stays on in the beginning of the on cycle. We observe that shortening the transmit antenna cycle to as little as two seconds does not significantly affect the average connection delays for the arriving clients. This is due to the time it takes for the client to detect the network and initiate the connection. Therefore we utilized the two seconds on time during the cycle for the transmit antenna to obtain the energy savings in Figure 11.

When clients are associated, power cycling can introduce delays into the application the user is interacting with. Figure 13 presents a cumulative distribution of the delays seen by the response to the first packet due to the transmit antenna

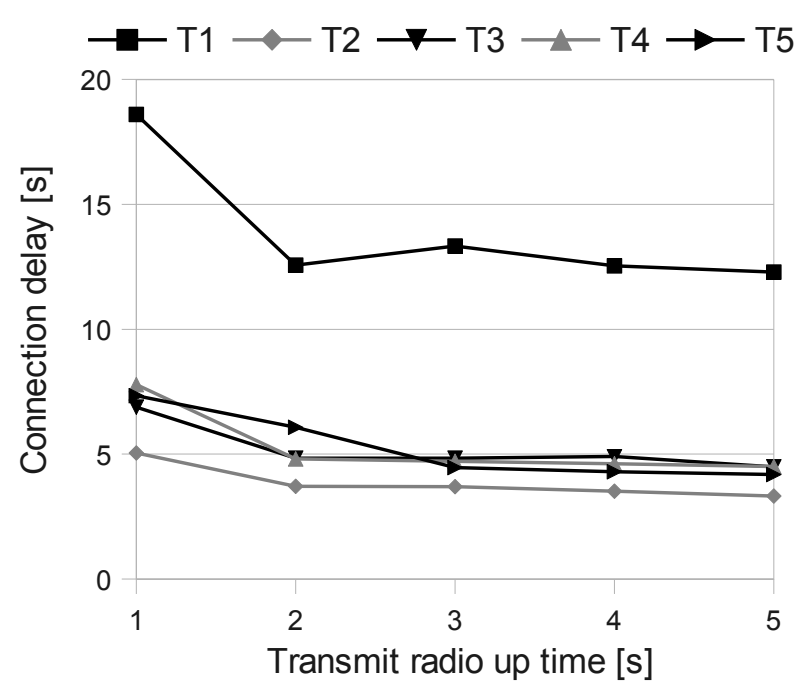

Fig. 12. Impact of decreased transmit antenna duty cycle on the association delays.

powering up. The transmit antenna takes $85 \mathrm{~ms}$ to transition to an active state and begin to serve traffic. As we can see from Figure 13, the majority of transmit antenna power ups can be fully overlapped by the time it takes for the packet to be serviced by the remote server before the router needs to reply to the client with the packet. The remaining delays will further be overlapped by the perception threshold and as a result the user will not be able to observe any additional delays. In addition, the delay is only encountered after a period of inactivity. Any subsequent activity that follows and requires high performance form the network (real time activity, large file transfers, etc.) will not see any performance degradation because any idle periods will be less than the break-even time required for the router to re-enter a low power mode.

Additional delays seen by the clients due to energy saving on the router will slightly decrease the energy efficiency of the client. This may be because the client transmits additional packets, or just has to keep its radios on for a longer period of time. However, the amount of time that a client may be in a less energy efficient state when compared to no special behavior on the router is limited to just a few seconds. Even with many clients, the amount of energy consumed in a nonoptimal state will not be as great as the energy saved by the router's energy saving actions.

\section{IMPLEMENTATION RESULTS}

The results thus far have been from replaying each network trace through a simulator under different situations while keeping track of additional introduced delay and calculating the resulting energy savings. Simulation is a valuable tool, but we also wanted to verify that our approach will work when implemented on real hardware. This will demonstrate the feasibility of our energy saving solution and validate our simulated results. We had selected the Asus RT-N16 for our evaluation because of its support by the Open Source DD- 


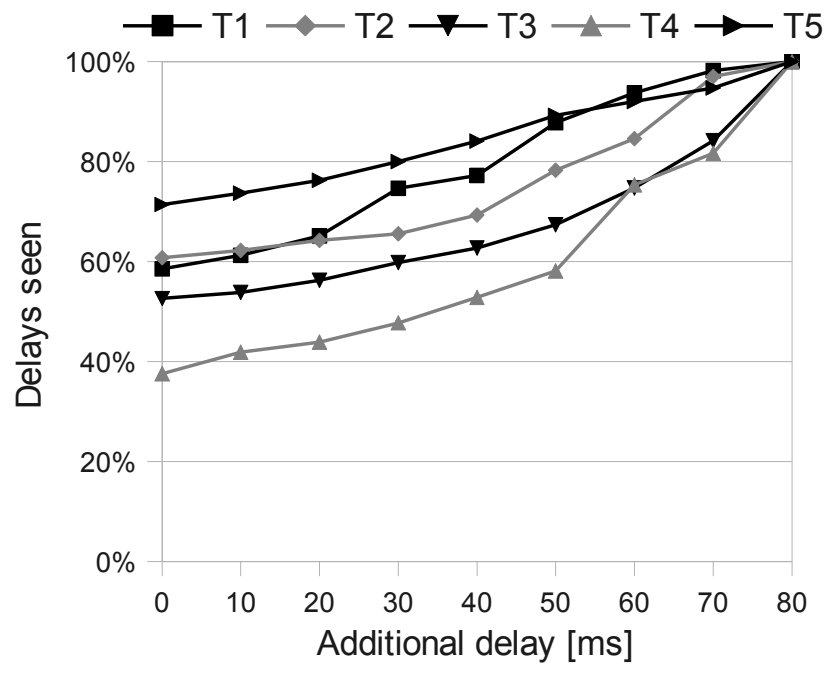

Fig. 13. Delay due to transmit antenna power ups on initial transmitted packets.

WRT firmware, and the source code is freely available for the firmware/driver modifications. However, the driver support for the radios was very limited, both in actual code and the required binary firmware, and did not offer the necessary support to execute fine-grained control of the transmit and receive functionality of the wireless chipset.

While we could not have the router actually transition between low power states, we were able to emulate this behavior. We wrote a small $\mathrm{C}$ program that interfaced with the Linux kernel's network configuration on the router to control packet loss and delay seen by network clients as if the router were actually turning its antennas on and off. The program follows the same logic previously described in deciding when to change the router's network state, but instead of issuing calls to control power the state of the radios rather introduces delay or loss packet in the router's network queue in a dynamic fashion.

The program uses the libnl-route library to interface with the Linux kernel's network routing control and leverages the kernel's network emulation module (netem) to add or remove the appropriate qdiscs to the wireless interface. Counts about transmitted and received packets were read from the wireless interface's RX and TX statistics available within the / sys / file system. Only the wireless interface was affected; wired ports were unchanged. Refer to Figure 14 for the setup of our test.

From a client's perspective, when the program was running the router would then be acting as if it were switching to a lower power mode when a sufficient period of idle time had elapsed, as previously described. This allowed us to test how clients respond in real life and verify that it is possible for a wireless router to dynamically switch to a lower power state without breaking existing clients. With the router now acting as if it were powering down its radios, we used our traces to generate identical traffic patterns between a laptop connected

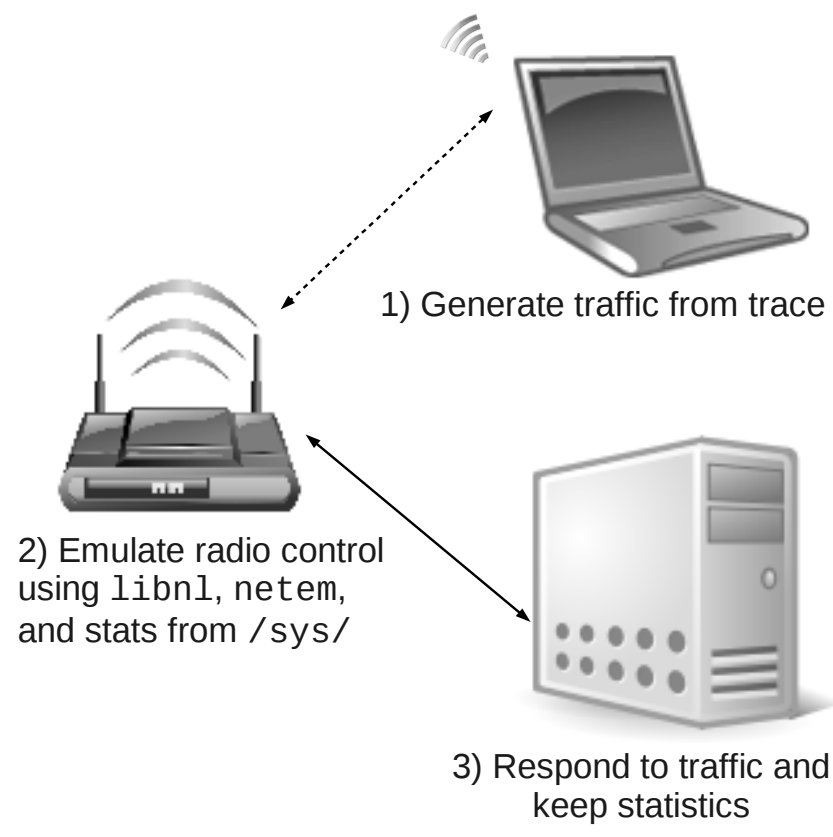

Fig. 14. Diagram of router implementation test configuration.

to the wireless network and another computer connected via a wired cable. This allowed us to measure both the additional delay that our solution introduces and the number of retransmitted packets due to timeouts higher up in the network stack.

All network communication has some inherent delay from the time a packet is sent and when the response is received. Figure 15 shows the total percent of time in each trace that was due to network delay, under normal operation and with power cycling emulation enabled on the router. With normal operation, at most $0.5 \%$ of all time in the trace was consumed in network delays, while power cycling raises this up to at most $1.75 \%$. In Traces 3 and 4 we observe largest increases; however even in those cases the delays will not be noticeable to the user because the individual delays are less than $85 \mathrm{~ms}$ as shown in Figure 13 and are beneath the perception threshold. Furthermore, real time applications do not experience this extra delay because the break-even timeout is never reached. Only non-interactive applications encounter the delay and they are able to tolerate it.

We also measured the amount of packet loss and retransmission due to timeouts or the router being in a state where it would drop incoming packets. There was not a significant increase in the number of retransmitted packets when comparing between normal operation and power cycling. Packets that were re-transmitted usually were from a long-lived TCP stream in the trace that was idle long enough for the router to enter a low power state before the application attempted to communicate again. However, this is exactly what TCP was designed to do over a potentially unreliable network, and so the application using TCP would not be aware of the duplicate packet transmission. 


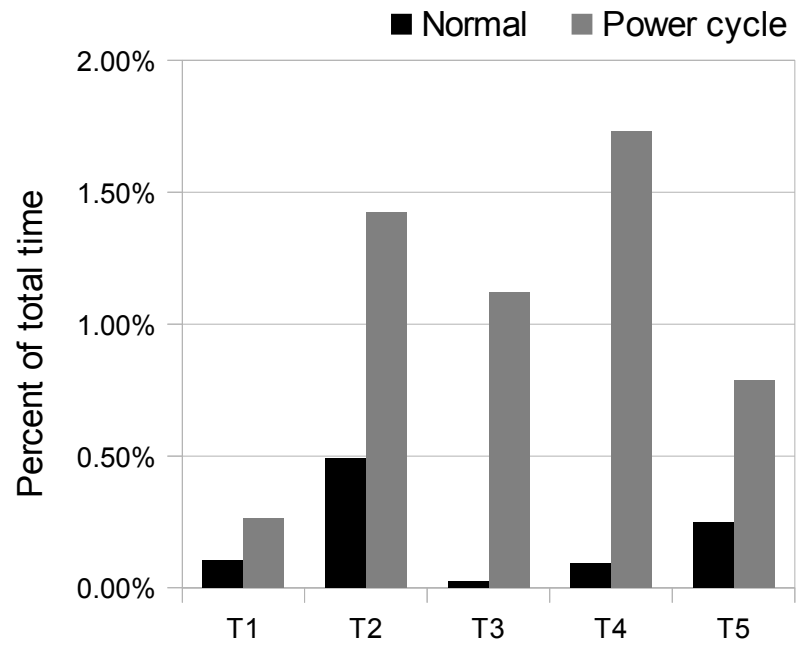

Fig. 15. Additional delay exposed to applications due to power cycling.

Figure 16 presents a comparison of our simulation and implementation approaches for the total number of times that the router would enter a lower power state when clients were associated to the wireless network. (This does not count periods of sleep when no clients were present, as described in Section III-B.) The results are normalized to the number of sleeps reported by the simulator, as some traces experienced significantly more sleep opportunities than others. In all traces, except T4, the number of observed times that the router would enter a lower power state increased an average of $5.8 \%$ when replaying the trace on the router itself. This would imply that the projected energy savings may be slightly better when running on actual hardware.

It should be pointed out that while replaying static traces can be useful to enable comparison with previous results, it is not a perfect representation of interactive traffic which would be seen on a live network. We do not see the dynamic response of an application retransmitting a packet and the subsequent changes in that application's network data caused by the router having transitioned to a low power state. However, this does allow us to demonstrate that a wireless client can continue to use a network employing this energy saving approach without requiring special configuration.

Our results of making changes to the operation of a home router to support the power cycling approach show that this approach is both feasible and can be performed without significantly impacting a user's experience when they wish to use their network. The logic to implement the energy saving approach is straight-forward and would be easy to include in a future firmware update for routers with appropriate hardware support.

Additionally, the periods of time that the router's antennas remained in the various power states was slightly better than the results of our simulation results above, which would imply that the energy consumption of our router would also be similar if the hardware allowed our fine-grained control. Our attempt

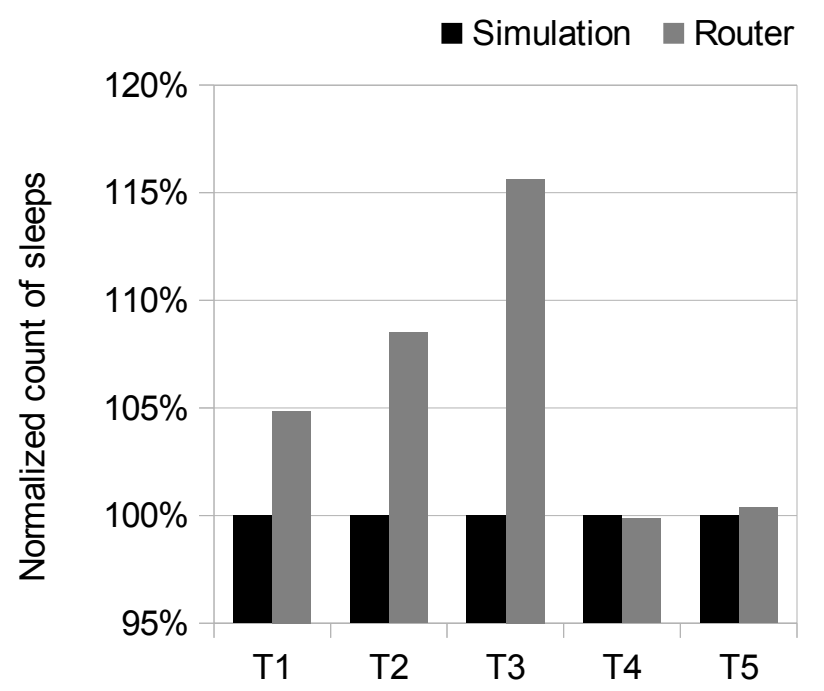

Fig. 16. Normalized number of sleeps per trace with active clients.

at implementing our energy saving solution in hardware shows that there is viable potential for our approach.

\section{FUTURE WORK}

While we were able to implement and successfully test the simultaneous power cycling of both transmit and receive antennas, we encountered significant challenges trying to decouple the transmit and receive antennas and control them individually on our router.

As previously mentioned, the Asus RT-N16 can run a Linux firmware, and source code is freely available. However, the open source driver support for the radios depends on a binary firmware which runs on the chip, and is not publicly available. We were unable to gain access to the source for this firmware from the chipset manufacturer. We then investigated another widely supported router, the Buffalo WZR-HP-G300NH-R WiFi-N, with the same outcome of not being able to penetrate the binary portions of the driver.

We were able to work around this issue somewhat via manipulation of the router's network queues to validate clients handling extra delays that would be seen if the router were running in a power saving mode, but this does not provide any insight into how much actual energy consumption would have been reduced. We are continuing to watch new generations of wireless routers to see if newer models have drivers that will allow us the fine-grained control that is necessary.

There are many variables which are heavily dependent on the actual router itself, such as the break-even time and transition times as the radios are powered on and off. This implies that each router would have to be analyzed before it could maximize energy savings. However, we would like to develop a simple model that would give optimal values when provided with key information about the router. This would not be hard to do given specifications from the manufacturer, 
and would allow this energy saving approach to be applied more widely.

As part of our future work on this project, we will investigate current routers that are more and more sophisticated, and we expect that manufacturers will include advanced power control due to increasing power demand of the home router. In addition, we are considering building a home router from available hardware components that will allow us to experiment with the proposed optimizations while waiting for manufacturers to implement advanced antenna control.

\section{RELATED WORK}

Home networks have become popular in current research activities due to their large deployment and relatively little understanding of the network patterns. Yin and Yang present a literature review of home network research trends in [14] which shows that from 1998 to 2009 there was an annual growth rate in home network research of about $20 \%$, which is likely due to the increase in home router use. Such research on home networks has been broad in focus, but can be categorized into a few areas. Work on traffic engineering, measurement, and general router attribute optimization have all been conducted.

Measuring the characteristics of home networks has been an active area of focus for researchers. They are trying to understand what is going on inside the home network [15], [16], as well as the performance of home networks [17], [18]. Additionally, error statistics have been collected [19] to aid in troubleshooting.

Some general optimization research has been conducted with the goal of making home networks more power efficient. Palem and Tozlu studied the energy consumption of two selected consumer grade access points with different hardware and software architectures [20]. Their study discusses the contribution of the components on the access point to the overall energy consumption. Park et al. [21] and Virolainen and Saaranen [22] have looked for solutions to lower the power use of home networks. Park et al. introduced a system that can effectively save energy by applying a small embedded system through remote control use on a smart phone, while Virolainen and Saaranen examined using UPnP low power which allows devices in power saving modes to be discovered and woken up.

Goma et al. propose in [23] an alternative approach to reducing the power consumption of home wireless networks. Their solution is to aggregate the traffic of many overlapping wireless networks onto a single network that possesses the necessary bandwidth and turn off all other networks. However, this solution requires the cooperation of both individuals and ISPs, and depends on a dense population of sparsely utilized wireless networks.

\section{CONCLUSION}

In this paper we analyzed several network traces from home users in an attempt to reduce the energy consumption of personal wireless routers. Using these traces we were able to show that most networks continue to see traffic, even when no wireless clients are present. This insight prompted a solution to reduce the amount of broadcast traffic transmitted over an idle wireless network to extend periods of idle time.

We also explored ways of controlling the state of the transmit and receive radios on the router, and of exploiting periods of idle time to put the router into an even lower power mode, even when clients are present on the network. Various methods were proposed, with the limitation that any changes to the operation of the router must not break compatibility with existing clients. The final solution was evaluated using both simulation and modification of an actual router to behave as if it were entering low power states. Projected wireless energy savings range from $12-59 \%$, depending on the network activity and method of saving energy.

\section{ACKNOWLEDGMENTS}

This research was based upon work supported by the National Science Foundation under Grants No. 0844569 and 1064963. Any opinions, findings, and conclusions or recommendations expressed in this material are those of the author(s) and do not necessarily reflect the views of the National Science Foundation.

\section{REFERENCES}

[1] M. Webb et al., "Smart 2020: Enabling the low carbon economy in the information age," The Climate Group. London, vol. 1, no. 1, pp. 1-1, 2008.

[2] National Telecommunications \& Information Administration, http://www.ntia.doc.gov/blog/2013/household-broadband-adoptionclimbs-724-percent.

[3] ASUS RT-AC66U 802.11ac Wireless-AC1750 Router Review, http://www.legitreviews.com/asus-rt-ac66u-802-11ac-wireless-ac1750router-review_2207/5.

[4] U.S. Energy Information Administration, http://www.eia.gov/tools/faqs/ faq.cfm?id=97\&t=3.

[5] S. Almekinders, http://us.hardware.info/reviews/2721/11/asus-rtn66u-review-lightning-fast-450mbps-router-test-results-energyconsumption.

[6] S. Nedevschi, L. Popa, G. Iannaccone, S. Ratnasamy, and D. Wetherall, "Reducing network energy consumption via sleeping and rateadaptation."

[7] CRAWDAD, http://www.crawdad.org/.

[8] DD-WRT Wiki, http://www.dd-wrt.com/wiki/index.php/Asus_RT-N16.

[9] F. I. Di Piazza, S. Mangione, and I. Tinnirello, "On the effects of transmit power control on the energy consumption of wifi network cards," in Quality of Service in Heterogeneous Networks. Springer, 2009, pp. 463-475.

[10] D. Halperin, B. Greenstein, A. Sheth, and D. Wetherall, "Demystifying 802.11 n power consumption," in Proceedings of the 2010 international conference on Power aware computing and systems. USENIX Association, 2010, p. 1.

[11] Documentation - Linux Wireless, http://wireless.kernel.org/en/ developers/Documentation.

[12] Link-Layer Retransmissions in IEEE $802.11 \mathrm{~g}$ based Industrial Networks, http://www.iiss.oeaw.ac.at/flexware/documents/publications/ 2010WFCS_Dominguez.pdf. 
[13] B. Shneiderman and S. Ben, Designing The User Interface: Strategies for Effective Human-Computer Interaction, 4/e (New Edition). Pearson Education India, 2003.

[14] C.-Y. Yin and J.-M. Yang, "A scientific assessment of home networking research trends," in Information Sciences and Interaction Sciences (ICIS), 2010 3rd International Conference on, 2010, pp. 421-425.

[15] S. Russ and S. Haghani, "Behavior of 802.11g traffic at high sustained bit rates in the home," in Consumer Electronics, 2009. ICCE '09. Digest of Technical Papers International Conference on, 2009, pp. 1-2.

[16] K. Xu, F. Wang, and M. Lee, "HomeTPS: Uncovering what is happening in home networks," in Consumer Communications and Networking Conference (CCNC), 2012 IEEE, 2012, pp. 40-41.

[17] I. Kofler, R. Kuschnig, and H. Hellwagner, "Evaluating the networking performance of Linux-based home router platforms for multimedia services," in Multimedia and Expo (ICME), 2011 IEEE International Conference on, 2011, pp. 1-6.

[18] S. Sundaresan, W. de Donato, N. Feamster, R. Teixeira, S. Crawford, and A. Pescapè, "Measuring home broadband performance," Commun. $A C M$, vol. 55, no. 11, pp. 100-109, Nov. 2012. [Online]. Available: http://doi.acm.org/10.1145/2366316.2366337

[19] A. Muller, G. Munz, and G. Carle, "Collecting router information for error diagnosis and troubleshooting in home networks," in Local Computer Networks (LCN), 2011 IEEE 36th Conference on, 2011, pp. 764-769.

[20] G. Palem and S. Tozlu, "On energy consumption of Wi-Fi access points," in Consumer Communications and Networking Conference (CCNC), 2012 IEEE, 2012, pp. 434-438.

[21] S.-H. Park, H.-J. Shin, M.-S. Kim, and C.-G. Kim, "Implementation of a low cost home energy saver based on OpenWRT," vol. 215 LNEE, Pyeong Chang, Korea, Republic of, 2013, pp. 761 - 765. [Online]. Available: http://dx.doi.org/10.1007/978-94-007-5860-5_91

[22] A. Virolainen and M. Saaranen, "Networked power management for home multimedia," in Consumer Communications and Networking Conference, 2008. CCNC 2008. 5th IEEE, 2008, pp. 331-332.

[23] E. Goma, M. Canini, A. Lopez Toledo, N. Laoutaris, D. Kostić, P. Rodriguez, R. Stanojević, and P. Yagüe Valentin, "Insomnia in the access: Or how to curb access network related energy consumption," SIGCOMM Comput. Commun. Rev., vol. 41, no. 4, pp. 338-349, Aug. 2011. [Online]. Available: http://doi.acm.org/10.1145/2043164.2018475 